\title{
GENERATING SETS AND A STRUCTURE OF THE WREATH PRODUCT OF GROUPS WITH NON-FAITHFUL GROUP ACTION
}

\author{
RUSLAN V. SKURATOVSKII ${ }^{1,2, *}$ \\ ${ }^{1}$ Faculty of Mathematics and Computer Science, NTUU, KPI named by "I. Sikorskiy", pr. Pobedy 37, \\ Kiev 03056, Ukraine \\ ${ }^{2}$ Faculty of Computer Science and Applied Mathematics, IAPM, Frometovskaiy 2, Kiev 03039, Ukraine
}

*Corresponding author: r.skuratovskii@kpi.ua, ruslan@unicyb.kiev.ua, ruslcomp@mail.ru

\begin{abstract}
Given a permutational wreath product sequence of cyclic groups, we investigate its minimal generating set, the minimal generating set for its commutator and some properties of its commutator subgroup. We generalize the result presented in the book of J. Meldrum [11] also the results of A. Woryna [4]. The quotient group of the restricted and unrestricted wreath product by its commutator is found. The generic sets of commutator of wreath product were investigated. The structure of wreath product with non-faithful group action is investigated. We strengthen the results from the author $[17,19]$ and construct the minimal generating set for the wreath product of both finite and infinite cyclic groups, in addition to the direct product of such groups. We generalise the results of Meldrum J. [11] about commutator subgroup of wreath products since, as well as considering regular wreath products, we consider those which are not regular (in the sense that the active group $\mathcal{A}$ does not have to act faithfully). The commutator of such a group, its minimal generating set and the center of such products has been investigated here. The minimal generating sets for new class of wreath-cyclic geometrical groups and for the commutator of the wreath product are found.
\end{abstract}

Received 2019-10-03; accepted 2019-11-20; published 2020-01-02.

2010 Mathematics Subject Classification. 20B27, 20E08, 20B22.

Key words and phrases. wreath product of group; minimal generating set of commutator subgroup of wreath product of groups; center of non regular wreath product; spherically homogeneous rooted tree; semidirect product.

(C)2020 Authors retain the copyrights of their papers, and all open access articles are distributed under the terms of the Creative Commons Attribution License. 


\section{IntroduCtion}

The form of commutator presentation [11] has been given here in the form of wreath recursion [10] and additionally, its commutator width has been studied. The results about commutators's structure given in [11] were improved.

Lucchini A. [6] previously investigated a case of the generating set of $C_{p}^{n-1} \prec G$, where $G$ denotes a finite $n$-generated group, $p$ is a prime which does not divide the order $|G|$ and $C_{p}$ denotes the cyclic group of order p. The results of Lucchini A. [6] tell us that the wreath product $C_{p}^{n-1}$ \& $G$ is also $n$-generated. We firstly consider the active group $G$ which is cyclic and then generalize this wreath product for both iterated wreath products and for the direct product of iterated wreath products of cyclic groups. It should be noted that to some extent a similar question for iterated wreath product was studied was studied by Bondarenko I [3]. One of the goal of our research is to study the center and commutator subgroup of wreath product with non-faithful action of active group on the set. Also as the goal of our paper is the minimal generating set and upper bound of minimal size of the generating set of the commutator subgroup of such class of group. The structure of center and quotient group by its commutator subgroup for a of such non-regular wreath product were still not investigated.

\section{Prelimenaries}

Let $G$ be a group. We denote by $d(G)$ the minimal number of generators of the group $G[3,6]$. The commutator width of $G$ [14], denoted $c w(G)$, is defined to be the least integer $n$, such that every element of $G^{\prime}$ is a product of at most $n$ commutators if such an integer exists, and otherwise is $c w(G)=\infty$.

The estimations of the upper bound of generating set of commutator subgroup were given by [14]. The property of commutator widths for groups and elements has proven to be important and in particular, its connections with stable commutator length and bounded cohomology has become significant.

Meldrum J. [11] briefly considered one form of commutators of the wreath product $A$ 々 $B$. In order to obtain a more detailed description of this form, we take into account the commutator width $(c w(G))$ as presented in work of Muranov A. [12].

The form of commutator presentation [11] has been given here in the form of wreath recursion [10] and additionally, its commutator width has been studied.

The subtree of $X^{*}($ or $\mathbb{T})$ which is induced by the set of vertices $\cup_{i=0}^{k} X^{i}$ is denoted by $X^{[k]}$ (or $\mathbb{T}_{k}$ ). Denote the restriction of the action of an automorphism $g \in A u t X^{*}$ to the subtree $X^{[l]}$ by $\left.g_{(v)}\right|_{X^{[l]}}$. It should be noted that a restriction $\left.g_{(v)}\right|_{X^{[1]}}$ is called the vertex permutation (v.p) of $g$ in a vertex $v$. 


\section{Minimal generating Set of Direct product of Wreath Products of CyClic Groups}

This work strengthens previous results by the author [17] and will additionally consider a new class of groups. This class is precisely the wreath-cyclic groups and will be denoted by $\Im$. Let $G \in \Im$, then this class is constructed by formula:

$$
G=\left(\underset{j_{0}=0}{\stackrel{n_{0}}{2}} C_{k_{j_{0}}}\right) \times\left(\underset{j_{1}=0}{\stackrel{n_{1}}{2}} C_{k_{j_{1}}}\right) \times \cdots \times\left(\underset{j_{l}=0}{\stackrel{n_{l}}{2}} C_{k_{j_{l}}}\right), 1 \leq k_{j_{i}}<\infty, n_{i}<\infty,
$$

where the orders of $C_{i_{j}}$ are denoted by $i_{j}$.

It should be noted that at the end of this product, a semidirect product could arise with a given homomorphism $\phi$, which is defined by a free action on the set $\mathbb{Z}$. In other words, one would obtain a group of the form $\left(\prod_{i=1}^{k} G_{i}\right)^{n} \ltimes_{\phi} \mathbb{Z}$.

Note that the last group here is isomorphic to one of the fundamental orbital groups $O_{f}(f)$ of the Morse function $f$. Namely, we have $\pi_{0}\left(S,\left.f\right|_{\partial M}\right)[21]$.

Consider now the group $H=\sum_{j=1}^{n} C_{i_{j}}$, whose orders $i_{j}$ for all $C_{i_{j}}$ are mutually coprime for all $j>1$ and whose number of cyclic factors in the wreath product is finite. We will call such group $H$ wreath-cyclic.

Note that the multiplication rule of automorphisms $g, h$ which are presented in the form of wreath recursion [13] $g=\left(g_{(1)}, g_{(2)}, \ldots, g_{(d)}\right) \sigma_{g}, h=\left(h_{(1)}, h_{(2)}, \ldots, h_{(d)}\right) \sigma_{h}$, is given precisely by the formula:

$$
g \cdot h=\left(g_{1} h_{\sigma_{g}(1)}, g_{2} h_{\sigma_{g}(2)}, \ldots, g_{d} h_{\sigma_{g}(d)}\right) \sigma_{g} \sigma_{h}
$$

In the general case, if an active group is not cyclic, then the cycle decomposition of an $n$-tuple for automorphism sections will induce the corresponding decomposition of the $\sigma_{g}$. If $\sigma$ is v.p of automorphism $g$ at $v_{i j}$ and all the vertex permutations below $v_{i j}$ are trivial, then we do not distinguish $\sigma$ from the section $g_{v_{i j}}$ of $g$ which is defined by it. That is to say, we can write $g_{v_{i j}}=\sigma=\left(v_{i j}\right) g$ as proposed by Bartholdi L., Grigorchuk R. and Šuni Z. [1].

We now make use of both rooted and directed automorphisms as introduced by Bartholdi L., Grigorchuk R. and Šuni Z. [1]. Recall that we denote a truncated tree by $\mathbb{T}$.

Definition 3.1. An automorphism of $\mathbb{T}$ is said to be rooted if all of its vertex permutations corresponding to non-empty words are trivial.

Let $l=x_{1} x_{2} x_{3} \cdots$ be an infinite ray in $\mathbb{T}$.

Definition 3.2. The automorphism $g$ of $\mathbb{T}$ is said to be directed along the infinite ray $l$ if all vertex permutations along $l$ and all vertex permutations corresponding to vertices whose distance to the ray $l$ is at least two are trivial. In such case, we say that $l$ is the spine of $g$ (as exemplified in Figure 1).

It should be noted that because we consider only truncated trees and truncated automorphisms here and for convenience, we will say rooted automorphism instead of truncated rooted automorphism. 
We reformulate and generalize the result of A. Woryna [4] about a minimal generating set of iterated wreath product. Also we make the statement more general after this theorem.

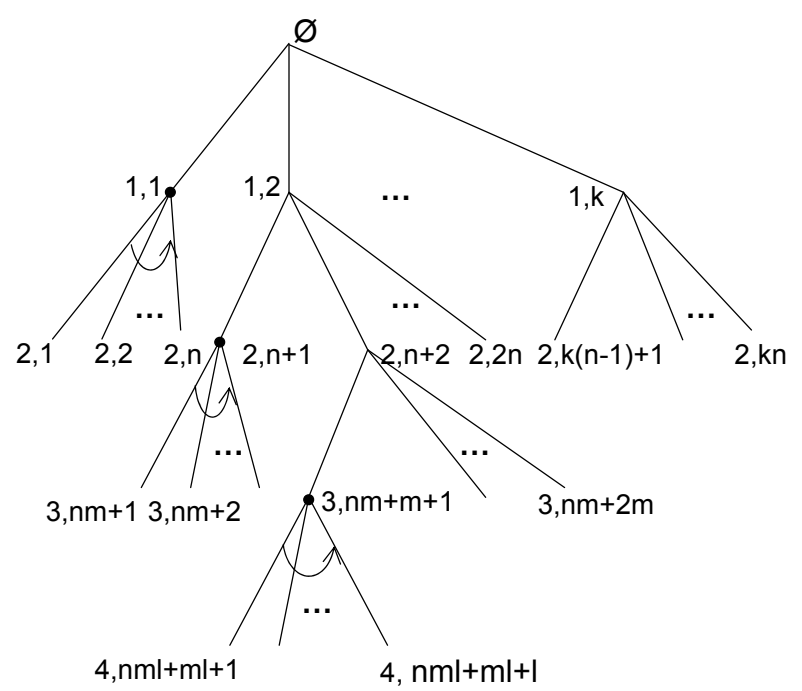

Fig. 1. Directed automorphism

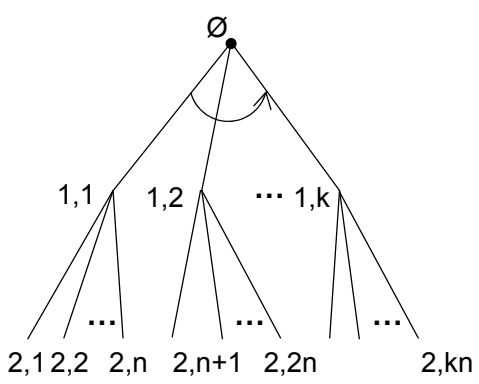

Fig. 2. Rooted automorhism

Theorem 3.1. If orders of cyclic groups $\mathbb{C}_{n_{i}}, \mathbb{C}_{n_{j}}$ are mutually coprime $i \neq j$, then the group $G=C_{i_{1}}$ ? $C_{i_{2}} \prec \cdots \prec C_{i_{m}}$ admits two generators, namely $\beta_{0}, \beta_{1}$.

Proof. Construct the generators of $\sum_{j=0}^{n} C_{i_{j}}$ as a rooted automorphism $\beta_{0}$ (Figure 2) and a directed automorphism $\beta_{1}[1]$ along a path $l$ (Figure 1) on a rooted labeled truncated tree $T_{X}$.

We consider the group $G=C_{i_{1}} \curlywedge C_{i_{2}} \prec \cdots \curlywedge C_{i_{m}}$. Construct the generating set of $C_{i_{1}} \prec C_{i_{2}} \prec \cdots \curlywedge C_{i_{m}}$, where the active group is on the left. Denote by $l c m_{1}=l c m\left(i_{2}, i_{3}, \ldots, i_{m}\right)$ the least common multiplier of the orders by $i_{2}, i_{3}, \ldots, i_{m}$. In a similar fashion, we denote

$$
l_{c m}=\operatorname{lcm}\left(i_{1}, i_{2}, \ldots, i_{k-1}, i_{k+1}, \ldots, i_{m}\right)
$$

similarly.

We utilise a presentation of those wreath product elements from a tableaux of Kaloujnine L. [9] which has the form $\sigma=\left[a_{1}, a_{2}(x), a_{3}\left(x_{1}, x_{2}\right), \ldots\right]$. Additionally, we use a subgroup of tableau with length $n$ which has the form

$$
\sigma_{(n)}=\left[a_{1}, a_{2}\left(x_{1}\right), \ldots a_{n}\left(x_{1}, \ldots, x_{n}\right)\right] .
$$

The tableaux which has first $n$ trivial coordinates was denoted in [20] by

$$
{ }^{(n)} \sigma=\left[e, \ldots, e, \alpha_{n+1}\left(x_{1}, \ldots, x_{n}\right), \alpha_{n+1}\left(x_{1}, \ldots, x_{n+1}\right), \ldots\right] .
$$


The canonical set of generators for the wreath product of $C_{p} \imath \cdots \imath C_{p} \prec C_{p}$ was used by Dmitruk Y. and Sushchanskii V. [7] and additionally utilized by the author [16]. This set has form

$$
\sigma^{\prime}{ }_{1}=\left[\pi_{1}, e, e, \ldots, e\right], \sigma^{\prime}{ }_{2}=\left[e_{1}, \pi_{2}, e, \ldots, e\right], \ldots, \sigma_{n}^{\prime}=\left[e_{1}, e, \ldots, e, \pi_{n}\right]
$$

We split such a tableau into sections with respect to (3.1), where the $i$-th section corresponds to portrait of $\alpha$ at $i$-th level. The first section corresponds to an active group and the crown of wreath product $G$, the second section is separated with a semicolon to a base of the wreath product. The sections of the base of wreath product are divided into parts by semicolon and these parts correspond to groups $C_{i_{j}}$ which form the base of wreath product. The $l$-th section of of a tableau presentation of automorphism $\beta_{1}$ corresponds to portrait of automorphism $\beta_{1}$ on level $X^{l}$.

The portrait of automorphisms $\beta_{1}$ on level $X^{l}$ is characterised by the sequence $\left(e, \ldots, e, \pi_{l}, e, \ldots, e\right)$, where coordinate $\pi_{l}$ is the vertex number of unique non trivial v.p on $X^{l}$, the sequence has $i_{0} i_{1} \ldots i_{l-1}$ coordinates. Therefore, our first generator has the form $\beta_{0}=\left[\pi_{1}, e, e, \ldots, e\right]$, which is the rooted automorphism. The second generator has the form

$$
\beta_{1}=[e ; \underbrace{\pi_{2}, e, e, \ldots, e}_{i_{1}} ; \underbrace{e_{e, e, e, e}^{i_{1} i_{2}}, \pi_{3}, e, \ldots, e}_{i_{2}} ; \underbrace{e_{e, \ldots, e}^{i_{2} i_{3}+i_{3}}, \pi_{4}, e, \ldots, e}_{i_{1} i_{2} i_{3}} ; e, \ldots, e],
$$

It should be noted that after the last (fourth) semicolon (or in other words before $\pi_{5}$ ) there are $i_{2} i_{3} i_{4}+$ $i_{3} i_{4}+i_{4}$ trivial coordinates. There are $i_{2} i_{3} i_{4} i_{5}+i_{3} i_{4} i_{5}+i_{4} i_{5}+i_{5}$ trivial coordinates before $\pi_{6}$ (or in other words after the fifth semicolon but before $\pi_{6}$ ). In a section after $k-1$ semicolon the coordinate of a nontrivial element $\pi_{k}$ is calculated in a similar way. We know from [20] that $\beta_{1}$ is generator of ${ }^{(2)} G$, i.e. 2 -base of $G$. Recall that ${ }^{(k)} G$ calls $k$-th base of $G$. The $\operatorname{subgroup~}{ }^{(k)} G$ is a subgroup of all tableaux of form ${ }^{(k)} u$ with $u \in G$.

Let $C_{n}=\left\langle\pi_{n}\right\rangle$ and set $\sigma_{1}=\beta_{0}$. We have to show that our generating set $\left\{\beta_{0}, \beta_{1}\right\}$ generates the whole canonical generating set. For this, we obtain the second new generator $\sigma_{2}$ in form of the tableau

$$
\sigma_{2}^{l c m_{2}}=\beta_{1}^{l c m_{2}}=[e ; \underbrace{\pi_{2}^{l c m_{2}}, e, e, \ldots, e}_{i_{1}} ; \underbrace{e, e, \ldots, e}_{i_{1} i_{2}} ; \underbrace{e, e, \ldots, e}_{i_{1} i_{2} i_{3}} ; e, \ldots, e] .
$$

Because $\operatorname{ord}\left(\pi_{1}\right)=i_{1}$ and $\left(i_{1}, l c m_{1}\right)=1$, we find that the element $\pi_{1}^{l c m_{1}}$ is generator of $C_{i_{1}}$ since $\operatorname{ord}\left(\pi_{1}\right)=\operatorname{ord}\left(\pi_{1}^{l c m_{1}}\right)$. We obtain that

$$
\sigma_{2}=\left(\beta_{1}^{l c m_{2}}\right)^{l c m_{2}^{-1}\left(\bmod i_{2}\right)}
$$

which corresponds to generator $\sigma_{2}$ of canonical generating set (3.1). Observe that $b_{3}=\sigma_{1}^{-1} \beta_{1}$ is generator of ${ }^{(3)} G$, i.e. it is precisely a 3 -base of $G$. 
It is known [20] that the generator $\sigma_{2}$ precisely generates the group that is isomorphic to the group $[U]_{2}$ for all 2-nd coordinate tableaux. From the same principle, one can obtain that

$$
\sigma_{3}=\beta_{1}^{l c m_{3}}=[e ; \underbrace{e, e, e, \ldots, e}_{i_{1}} ; \overbrace{\underbrace{e, e, \ldots, e}_{i_{2}}, \pi_{3}^{l c m_{3}}, e, \ldots, e}^{i_{1} i_{2}} ; \underbrace{\overbrace{e, \ldots, e, e, e, \ldots, e}^{i_{2} i_{3}}}_{i_{1} i_{2} i_{3}} ; e \ldots e] .
$$

This generator $\sigma_{3}$ generates the group which is isomorphic to the group of all $\left(2 i_{1}+2\right)$-th coordinate tableaux, which is precisely $[U]_{2 i_{1}+2}[20]$. Making use of the same principle allows us to express all the $\sigma_{i}$ from our canonical generating set.

Note that if it were a self-similar group, then it would be more useful to present it in terms of wreath recursion, as the set where $\beta_{0}$ is the rooted automorphism. Given a permutational representation of $C_{i_{j}}$ we can present our group by wreath recursion. We present $\beta_{1}$ by wreath recursion as $\beta_{1}=\left(\pi_{2}, \beta_{2}, e, e, \ldots, e\right)$. It would be written in form $\sigma_{1}^{l c m_{2}}=\beta_{1}^{l c m_{2}}=\left(\pi_{2}^{l c m_{2}}, \beta_{2}^{l c m_{2}}, e, e, \ldots, e\right)=\left(\pi_{2}^{l c m(2)}, e, e, \ldots, e\right)$, since $\operatorname{ord}\left(\pi_{2}\right)=i_{2}$ and $\left(i_{2}, l c m_{2}\right)=1$ then the element $\pi_{2}^{l c m_{2}}$ is generator of $C_{i_{2}}$ too, because $\operatorname{ord}\left(\pi_{2}\right)=\operatorname{ord}\left(\pi_{2}^{l c m_{2}}\right)$.

We then obtain the second generator $\sigma_{2}$ of canonical generating set by exponentiation $\left(\beta_{1}^{l c m_{2}}\right)^{l c m_{2}^{-1}\left(\bmod _{2}\right)}=\left(\pi_{2}, e, \ldots, e\right)$. Since we have obtained $\sigma_{2}=\left(\pi_{2}, e, \ldots, e\right)$, we can express $\sigma_{2}^{-1}=$ $\left(\pi_{2}^{-1}, e, \ldots, e\right)$, where $\pi_{2}$ is a state of $\sigma_{2}$.

Consider an alternative recursive constructed generating set which consists of nested automorphism $\beta_{1}$ states which are $\beta_{2}, \beta_{3}, \ldots, \beta_{m}$ and the automorphism $\beta_{0}$. The state $\beta_{2}$ is expressed as follows $\sigma_{2}^{-1} \beta_{1}=$ $\left(e, \beta_{2}, e, \ldots, e\right)$.

It should be noted that a second generator of a recursive generating set could be constructed in an other way, namely

$$
\beta_{2}^{\prime}=\beta_{1}{ }^{i_{2}}=\left(\pi_{2}{ }^{i_{2}}, \beta_{2}{ }^{i_{2}}, e, e, \ldots, e\right)=\left(e, \beta_{2}{ }^{i_{2}}, \ldots, e, e\right),
$$

where $\beta_{2}$ is the state in a vertex of the second level $X^{2}$.

We can then express the next state $\beta_{2}$ of $\beta_{1}$ by multiplying $\sigma_{2}^{-1} \beta_{1}=\left(e, \beta_{2}, e, \ldots, e\right)$. Therefore, by a recursive approach, we obtain $\beta_{2}=\left(\pi_{3}, \beta_{3}, e, \ldots, e\right)$ and analogously we obtain $\beta_{2}^{l c m_{3}}=\sigma_{3}^{l c m_{3}}=\left(\pi_{3}^{l c m_{3}}, e, \ldots, e\right)$. Similarly, we obtain

$$
\beta_{k-1}^{l c m_{k}}=\sigma_{k}^{l c m_{k}}=\left(\pi_{k}^{l c m_{k}}, e, \ldots, e\right)
$$

and $\sigma_{k}=\left(\beta_{k-1}^{l c m_{k}}\right)^{l c m_{k}^{-1}\left(\bmod i_{k}\right)}=\left(\pi_{k}, e, \ldots, e\right)$. The $k$-th generator of the recursive generating set can therefore be expressed as $\sigma_{k}^{-1} \beta_{k-1}=\left(e, \beta_{k}, e, \ldots, e\right)$.

The last generator of our generating set has another structure, namely $\sigma_{m}=\left(\pi_{m}, e, \ldots, e\right)$ which concludes the proof.

Let $\sum_{j=0}^{n} C_{i_{j}}$ be generated by $\beta_{0}$ and $\beta_{1}$ and $\sum_{l=0}^{m} C_{k_{l}}=\left\langle\alpha_{0}, \alpha_{1}\right\rangle$. Denote an order of $g$ by $|g|$. 
Theorem 3.2. If $\left(\left|\alpha_{0}\right|,\left|\beta_{0}\right|\right)=1$ and $\left(\left|\alpha_{1}\right|,\left|\beta_{1}\right|\right)=1$ or $\left(\left|\alpha_{0}\right|,\left|\beta_{1}\right|\right)=1$ and $\left(\left|\alpha_{1}\right|,\left|\beta_{0}\right|\right)=1$, then there exists a generating set of 2 elements for the wreath-cyclic group

$$
G=\left(\sum_{j=0}^{n} C_{i_{j}}\right) \times\left(\sum_{l=0}^{m} C_{k_{l}}\right)
$$

where $i_{j}$ are orders of $C_{i_{j}}$.

Proof. The generators $\alpha_{1}$ and $\beta_{1}$ are directed automorphisms, $\alpha_{0}, \beta_{0}$ are rooted automorphisms [1]. The structure of tableaux are described above in Theorem 1. In case $\left(\left|\alpha_{0}\right|,\left|\beta_{0}\right|\right)=1$ are mutually coprime and $\left(\left|\alpha_{1}\right|,\left|\beta_{1}\right|\right)=1$ are mutually coprime, then we group generator $\alpha_{0}$ and $\beta_{0}$ in vector that is first generator

of direct product $\left(\sum_{j=0}^{n} C_{i_{j}}\right) \times\left(\sum_{l=0}^{m} C_{k_{l}}\right)$. Therefore, the first generator of $G$ has form $\left(\alpha_{0}, \beta_{0}\right)$ and the second generator has form of vector $\left(\beta_{1}, \alpha_{1}\right)$. The generator $\alpha_{1}$ has a similar structure.

In order to express the generator $\sigma_{2}$ of the canonical set (3.1) from $\left\langle\alpha_{0}, \beta_{1}\right\rangle$ we change the exponent from $\beta_{1}$ to $l c m_{2}$. Analogously, we obtain $\sigma_{k}=\beta_{1}{ }^{l c m_{k}}$ which concludes the proof.

\section{Center and commutator subgroup of Wreath product their minimal generating Sets}

Let us find upper bound of generators number for $G^{\prime}$. Let $\mathcal{A}$ be a group and $\mathcal{B}$ a permutation group, i.e. a group $\mathcal{A}$ acting upon a set $X$, where the active group $\mathcal{A}$ can act not faithfully. Consider the set of all pairs $\{(a, f), f: X \rightarrow h, a \in \mathcal{A}\}$. We define a product on this set as

$$
\left\{\left(a_{1}, f_{1}\right)\left(a_{2}, f_{2}\right):=\left(a_{1} a_{2}, f_{1} f_{2}^{a_{1}}\right)\right\}
$$

where $f_{1}^{a_{2}}(x)=f_{1}\left(a_{2}(x)\right)$.

Theorem 4.1. If $W=(\mathcal{A}, X) \curlywedge(\mathcal{B}, Y)$, where $|X|=n,|Y|=m$ and active group $\mathcal{A}$ acts on $X$ transitively, then

$$
d\left(W^{\prime}\right) \leq(n-1) d(\mathcal{B})+d\left(\mathcal{B}^{\prime}\right)+d\left(\mathcal{A}^{\prime}\right)
$$

Proof. The generators of $W^{\prime}$ in form of tableaux [2]: $a^{\prime}{ }_{i}=\left(a_{i} ; e, e, e, \ldots, e\right), t_{1}=\left(e ; h_{j_{1}}, e, e, \ldots, c_{j_{1}}\right), \ldots$, $t_{k}=\left(e ; e, e, e, \ldots, h_{j_{k}}, e, \ldots, c_{j_{k}}\right), t_{l}=\left(e ; e, e, e, \ldots, h_{j_{l}}, c_{j_{l}}\right)$, where $h_{j}, c_{j_{l}} \in S_{B}, \mathcal{B}=\left\langle S_{B}\right\rangle, a_{i} \in S_{A}$, $A=\left\langle S_{A}\right\rangle$. Note that, on a each coordinate of tableau, that presents a commutator of $\left[a ; h_{1}, \ldots, h_{n}\right]$ and $\left[b ; g_{1}, \ldots, g_{n}\right], a, b \in \mathcal{A}, h_{i}, g_{j} \in \mathcal{B}$ can be product of form $a_{1} a_{2} a_{1}^{-1} a_{2}^{-1} \in \mathcal{A}^{\prime}$ and $h_{i} g_{a(i)} h_{a b(i)}^{-1} g_{a b a^{-1}(i)}^{-1} \in \mathcal{B}$, according to Corollary 4.9 [11]. This products should satisfy the following condition:

$$
\prod_{i \in X}^{n} h_{i} g_{a(i)} h_{a b(i)}^{-1} g_{a b a^{-1}(i)}^{-1} \in \mathcal{B}^{\prime} .
$$

That is to say that the product of coordinates of wreath product base is an element of commutator $\mathcal{B}^{\prime}$. As it was described above it is subdirect product of $\underbrace{\mathcal{B} \times \mathcal{B} \times \cdots \times \mathcal{B}}_{n}$ with the additional condition (4.1). This 
is the case because not all element of the subdirect product are independent because the elements must be chosen in such a way that (4.1) holds. We may rearrange the factors in the product in the following way:

$$
\prod_{i=1}^{n} h_{i} g_{a(i)} h_{a b(i)}^{-1} g_{a b a^{-1}(i)}^{-1}=\left(\prod_{i=1}^{n} h_{i} g_{i} h_{i}^{-1} g_{i}^{-1}\right)[g, h] \in \mathcal{B}^{\prime}
$$

where $[g, h]$ is a commutator in case $c w(B)=1$. We express this element from $\mathcal{B}^{\prime}$ as commutator $[g, h]$ if $c w(B)=1$. In the general case, we would have $\prod_{j=1}^{c w(B)}\left[g_{j}, h_{j}\right]$ instead of this element. This commutator are formed as product of commutators of rearranged elements of $\prod_{i=1}^{n} h_{i} g_{a(i)} h_{a b(i)}^{-1} g_{a b a^{-1}(i)}^{-1}$. Therefore, we have a subdirect product of $n$ the copies of the group $B$ which has been equipped by condition (4.1). The multiplier $c w(B)$

$\prod_{j=1}^{c}\left[g_{j}, h_{j}\right]$ from $\mathcal{B}^{\prime}$, which has at least $d\left(\mathcal{B}^{\prime}\right)$ generators

$$
\prod_{i=1}^{n} h_{i} g_{a(i)} h_{a b(i)}^{-1} g_{a b a^{-1}(i)}^{-1}=\left(\prod_{i=1}^{n} h_{i} g_{i} h_{i}^{-1} g_{i}^{-1}\right) \prod_{j=1}^{c w(B)}\left[g_{j}, h_{j}\right] \in \mathcal{B}^{\prime}
$$

Since $\left(\prod_{i=1}^{n} h_{i} g_{i} h_{i}^{-1} g_{i}^{-1}\right)=e$ and the product $\prod_{j=1}^{c w(B)}\left[g_{j}, h_{j}\right]$ belongs to $\mathcal{B}^{\prime}$, then condition $(4.1)$ holds. The assertion of a theorem on a recursive principle is easily generalized on multiple wreath product of groups.

Thus minimal total amount consists of at least $d\left(\mathcal{B}^{\prime}\right)$ generators for $n-1$ factors of group $\mathcal{B}, d\left(\mathcal{B}^{\prime}\right)$ generators for the dependent factor from $\mathcal{B}^{\prime}$ and $d(\mathcal{A})$ generators of the group $\mathcal{A}$.

It should be noted that not all the elements of commutator subgroup, that has structure of the subdirect product, are independent by (4.1), at least one of them must be chosen carefully such that would be (4.1) satisfied. This implies the estimation $d\left(W^{\prime}\right) \leq(n-1) d(B)+d\left(B^{\prime}\right)$.

Thus minimal total amount consists of at least $d\left(\mathcal{B}^{\prime}\right)$ generators for $n-1$ factors of group $\mathcal{B}, d\left(\mathcal{B}^{\prime}\right)$ generators for the dependent factor from $\mathcal{B}^{\prime}$ and $d(\mathcal{A})$ generators of the group $\mathcal{A}$ which concludes the proof.

We shall consider special case when a passive group $(\mathcal{B}, Y)$ of $W$ is a perfect group. Since we obtain a direct product of $n-1$ the copies of the group $B$ then according to Corollary 3.2. of Wiegold J. [22] $d\left(\mathcal{B}^{n}\right) \leq d(\mathcal{B})+n-1[22]$. More exact upper bound give us Theorem A. [22], which use $s$ a the size of the smallest simple image of $G$.

Therefore, in this case our upper bound has the form

$$
d\left(W^{\prime}\right) \leq \operatorname{cog}_{s} n+d\left(\mathcal{B}^{\prime}\right)+d\left(\mathcal{A}^{\prime}\right)
$$

Now we consider non-regular wreath product, where active group can be both as infinite as finite and consider a center of such group. We generalize a result of Meldrum J. [11] because we consider not only the permutation wreath product groups, but the group $\mathcal{A}$ does not have to act on the set $X$ faithfully, hence 
$(\mathcal{A}, X) \prec \mathcal{B}$ is not regular wreath product, where $\mathcal{B}$ is a passive group. Recall that an action is said to be faithful if for every $g \in G$, there exists $x$ from $G$-space $X$ such that $x^{g} \neq x$.

Let $X=\left\{x_{1}, x_{2}, \ldots, x_{n}\right\}$ be $\mathcal{A}$-space. If an non faithfully action by conjugation determines a shift of copies of $\mathcal{B}$ from direct product $\mathcal{B}^{n}$ then we have not standard wreath product $(\mathcal{A}, X)$ $\mathcal{B}$ that is semidirect product of $\mathcal{A}$ and $\prod_{x_{i} \in X} \mathbb{B}$ that is $\mathcal{A} \ltimes_{\varphi}(\mathcal{B})^{n}$ and the following Proposition holds. Let $\mathcal{K}=\operatorname{ker}(\mathcal{A}, X)$ that is subgroup of $\mathcal{A}$ that acts on $X$ as a pointwise stabilizer, that is kernel of action of $\mathcal{A}$ on $X$.

Denote by $Z(\tilde{\triangle}(\mathcal{B}))$ the subgroup of diagonal [5] Fun $(X, Z(B))$ of functions $f: X \rightarrow Z(B)$ which are constant on each orbit of action of $A$ on $X$ for unrestricted wreath product, and denote by $Z\left(\triangle\left(\mathcal{B}^{n}\right)\right)$ the subgroup of diagonal Fun $\left(X, Z\left(\mathcal{B}^{n}\right)\right)$ of functions with the same property for restricted wreath product, where $n$ is number of non-trivial coordinates in base of wreath product.

Proposition 4.1. A center of the group $(\mathcal{A}, X) \curlywedge \mathcal{B}$ is direct product of normal closure of center of diagonal of $Z\left(\mathcal{B}^{n}\right)$ i.e. $\left(E \times Z\left(\triangle\left(\mathcal{B}^{n}\right)\right)\right)$, trivial an element, and intersection of $(\mathcal{K}) \times E$ with $Z(\mathcal{A})$. In other words,

$$
Z((\mathcal{A}, X) \curlywedge \mathcal{B})=\langle(1 ; \underbrace{h, h, \ldots, h}_{n}), e, Z(\mathcal{K}, X) \curlywedge \mathcal{E}\rangle \simeq\langle Z(\mathcal{A}) \cap \mathcal{K}) \times Z\left(\triangle\left(\mathcal{B}^{n}\right)\right\rangle
$$

where $h \in Z(\mathcal{B}),|X|=n$.

For restricted wreath product with $n$ non-trivial coordinate: $Z((\mathcal{A}, X) \curlywedge \mathcal{B})=$ $\langle(1 ; \ldots, h, h, \ldots, h, \ldots), e, Z(\mathcal{K}, X) \imath \mathcal{E}\rangle \simeq(Z(\mathcal{A}) \cap \mathcal{K}) \times Z\left(\triangle\left(\mathcal{B}^{n}\right)\right)$.

In case of unrestricted wreath product we have: $Z((\mathcal{A}, X) \curlywedge \mathcal{B})=$ $\left\langle\left(1 ; \ldots, h_{-1}, h_{0}, h_{1}, \ldots, h_{i}, h_{i+1}, \ldots,\right), e, Z(\mathcal{K}, X) \imath \mathcal{E}\right\rangle \simeq(Z(\mathcal{A}) \cap \mathcal{K}) \times Z(\tilde{\triangle}(\mathcal{B}))$.

Proof. The elements of center subgroup have to satisfy the condition: $f: X \rightarrow B$ such is constant on each orbit $\mathcal{O}_{j}$ of action $\mathcal{A}$ on $X$ i.e. $f(x)=b_{i}$ for any $x \in \mathcal{O}_{j}$. Also every $b_{x}: b_{x} \in Z(\mathcal{B})$. Indeed the elements of form $(1 ; \underbrace{h, h, \ldots, h}_{n})$ will not be changed by action of conjugation of any element from $\mathcal{A}$ because any permutation elements coordinate of diagonal of $\mathcal{B}^{n}$ does not change it. Also $h$ commutes with any element of base of $(\mathcal{A}, X)$ 乙 $\mathcal{B}$ because $h$ from centre of $\mathcal{B}$. Since the action is defined by shift on finite set $X,|X|=n$ is not faithfully, then its kernel $\mathcal{K} \neq E$ which confirms the proposition. Also elements of $\operatorname{subgroup}(\mathcal{A}, X) \mathcal{L})$ belongs to $Z((\mathcal{A}, X) \curlywedge \mathcal{B})$ iff it acts trivial on $X$.

This is generalization of Theorem 4.2 from the book [11] because action of $\mathcal{A}$ is not faithfully.

Example 4.1. If $\mathcal{A}=\mathbb{Z}$ then a centre $Z((\mathbb{Z}, X) \curlywedge \mathcal{B})=$

$\left\langle(1 ; \underbrace{h, h, \ldots, h}_{n}), e, n \mathbb{Z} \ltimes \mathcal{E}: h \in Z\left(\triangle\left(\mathcal{B}^{n}\right)\right)\right\rangle$. Since the action defined by shift on finite set $X$ is not faithfully, and its kernel is isomorphic to $n \mathbb{Z}$ because cyclic shift on $n$ coordinates is invariant on $X$. 
Generating set for commutator subgroup $\left(\mathbb{Z}_{n} \prec \mathbb{Z}_{m}\right)^{\prime}$, where $\mathbb{Z}_{n}, \mathbb{Z}_{m}$ have presentation in additive form, is the following:

$$
\begin{gathered}
h_{1}=(0 ; 1,0, \ldots, m-1), \\
h_{2}=(0 ; 0,1,0, \ldots, m-1), \\
\vdots \\
h_{n-1}=(0 ; 0, \ldots, 1, m-1) .
\end{gathered}
$$

Thus, it consist of $n$ tableaux of form $h_{i}=\left(h_{i 1}, \ldots, h_{i m}\right)$ and relations for coordinate of any tableau $h_{i}, i \in$ $\{1, \ldots, n-1\}$ is

$$
h_{i 1}+\cdots+h_{i n-1} \equiv 0(\bmod m)
$$

According to Theorem 3, for wreath product of abelian groups presented in multiplicative form, this relation has the form

$$
\prod_{i=1}^{n} h_{i} f_{i_{\pi_{a}}} h_{i_{\pi_{a} \pi_{b}}}^{-1} f_{i_{\pi_{a} \pi_{b} \pi_{a}^{-1}}}^{-1}[h, f]=\prod_{i=1}^{n}\left(h_{i} f_{i_{\pi_{a}}} h_{i_{\pi_{a} \pi_{b}}}^{-1} f_{i_{\pi_{a} \pi_{b} \pi_{a}^{-1}}^{-1}}^{-1} \prod_{j=i+1}^{i+2}\left[h_{j}, f_{j_{\pi_{a}}}\right]\right)=e .
$$

Example 4.2. If $G=\mathbb{Z}_{n} \curlywedge \mathbb{Z}_{m}$ is standard wreath product, then $d\left(G^{\prime}\right)=n-1$.

Let $G=Z \imath_{X} Z$ and $G=A \imath_{X} B$ be a restricted wreath product, where only $n$ non-trivial elements in coordinates of base of wreath product which are indexed by elements from $X$, in degenerated case $|X|=n$. $Z$ acts on $X$ by left shift. Also $A$ acts transitively from left.

Remark 4.1. The quotient group of a restricted wreath products $G=Z \imath_{X} Z$ by a commutator subgroup is isomorphic to $\mathbb{Z} \times \mathbb{Z}$. In previous conditions if $G=A \chi_{X} B$ then, $G / G^{\prime}=A / A^{\prime} \times B / B^{\prime}$. If $G=Z_{n}$ భ $Z_{m}$, where $(m, n)=1$, then $d\left(G / G^{\prime}\right)=1$. If $G=Z \imath Z$ is an unrestricted regular wreath product then $G / G^{\prime} \simeq Z \times E \simeq Z$.

Proof. Consider the element of $G=A \imath_{X} \mathcal{B}$, where $A$ can be $Z$ which acts on $X$ by left shift, then elements of commutator subgroup has form:

$\left[e ; \ldots, h_{-n}, \ldots, h_{0}, h_{1}, \ldots, h_{n}, \ldots,\right]$, where $h_{i} \in B$. According to Corollary 4.9 [11] the commutator of elements $h=\left[a ; h_{1}, \ldots, h_{n}\right], g=\left[b ; g_{1}, \ldots, g_{n}\right], g, h \in G$ satisfies the condition (4.1), which for case where $B$ is abelian such: $\prod_{i=1}^{n} h_{i} g_{a(i)} h_{a b(i)}^{-1} g_{a b a^{-1}(i)}^{-1}=e$, where $g_{i}, h_{i}$ are non trivial coordinates from base of group, $a, b \in A, g_{i}, h_{j} \in B$. The commutator with the shifted coordinate $h_{i} g_{a(i)} h_{a b(i)}^{-1} g_{a b a^{-1}(i)}^{-1}$ appears within the $i$-th coordinate position due to action of $A$. According to Corollary 4.9 [11] the set of elements satisfying condition (4.1) forms a commutator. Also the equivalent condition can be formulated:

$$
\prod_{i=1}^{n} h_{i} g_{i} h_{i}^{-1} g_{i}^{-1} \in \mathcal{B}^{\prime}
$$


Therefore, if $\mathcal{B}$ is abelian an element $h$ of $G$ belongs to $G^{\prime}$ iff $h$ satisfy a condition: $\prod_{i=1}^{n} h_{i}=e$.

For unrestricted wreath product to show that all base of wreath product is in the commutator subgroup we choose an element $\left[e ; \ldots, h_{-1}, h_{0}, h_{1}, \ldots\right]$, where $h_{i}$ is variable, and form a commutator which is an arbitrary element $\left[e ; \ldots, g_{-1}, g_{0}, g_{1}, \ldots\right]$ of wreath product base:

$\left[e ; \ldots, h_{-1}, h_{0}, h_{1}, \ldots\right][\sigma ; e, e, \ldots, e]\left[e ; \ldots, h_{-1}^{-1}, h_{0}^{-1}, h_{1}^{-1}, \ldots\right]\left[\sigma^{-1} ; e, e, \ldots, e\right]=$ $=\left[e ; \ldots, g_{-1}, g_{0}, g_{1}, \ldots\right]$. For convenience we present $Z$ in additive form. Then to previous equality holds the following equations have to be satisfied: $h_{0}-h_{1}=g_{0}, h_{1}-h_{2}=0, h_{2}-h_{3}=0, \ldots$ it implies that $h_{1}=h_{0}-1, h_{2}=h_{1}, h_{3}=h_{2}, \ldots h_{i}+1=h_{i}$. Therefore $h_{i}=0, i \geq 1$. From other side we have $h_{-1}-h_{0}=g_{0}, h_{-1}-h_{-2}=0, h_{-2}-h_{-3}=0, \ldots$ so $h_{-i}=g_{0}$, for all $i<0$. That is impossible in the restricted case but possible in the unrestricted. As a corollary $G / G^{\prime} \simeq Z \times Z$ for restricted case. Thus, for unrestricted case all base of $G$ is in $G^{\prime}$ as a corollary $G / G^{\prime} \simeq Z \times E$.

Thus, this group is a subdirect product of $\underbrace{B \times B \times \cdots \times B}_{n}$ with the additional condition (4.2) where, because for any element of the subgroup of coordinates there exists a surjective homomorphism acting upon $B$, we can conclude that $G^{\prime}$ must be a subdirect product. The commutator subgroup is the kernel of homomorphism $\varphi: G \rightarrow G / G^{\prime}$. More precisely,

$$
G=(Z, X) \curlywedge(Z, Y) \rightarrow G / G^{\prime} \simeq Z / Z^{\prime} \times Z / Z^{\prime}=\mathbb{Z} \times \mathbb{Z}
$$

In case $G=A \curlywedge \mathcal{B}$ the $\operatorname{ker} \varphi$ has the same structure, the homomorphism $\varphi$ maps those elements of $B^{n}$, as base of $G$, which satisfy $\prod_{i=1}^{n} h_{i}=e$, i.e. the elements of $B^{\prime}$ in $e$ of the group $G / G^{\prime}$. Thus, ker $\varphi=G^{\prime}$. To show that the properties of injectivity and surjectivity hold for this homomorphism, we chose the elements from $G$ which have the form $[e ; e, \ldots e, h, e, \ldots, e]$ that can be generator in canonical form of generating set of wreath product (3.1), where $h \notin G^{\prime}$, corresponding to a a specimen from the quotient group $B / B^{\prime}$. Also we chose independently, an element of the form $[a ; e, \ldots, e, \ldots, e]$ corresponding to a specimen of the quotient group $A / A^{\prime}$. Therefore, we must have a one-to-one correspondence between $G / G^{\prime}$ and $A / A^{\prime} \times B / B^{\prime}$. In this case, we obtain $G / G^{\prime} \simeq\left[A / A^{\prime} \times{ }^{B} / B^{\prime}\right]$. The basic property of homomorphism for generators in canonical form (3.1) is obviously accomplished.

In the scenario when the action of $Z$ upon the $n$ elements from the set is isomorphic to the action of $Z_{n}$ elements on the set or the action of the $Z_{n}$ elements on itself. In case $G=Z$ 々 $Z$ we have ${ }^{G} / G^{\prime} \simeq[\mathbb{Z} \times \mathbb{Z}]$.

For the group $G=Z_{n} \prec Z_{m}$ the same is true with $G / G^{\prime} \simeq\left[\mathbb{Z}_{n} \times \mathbb{Z}_{m}\right]$ and dependently of fact of $(m, n)=1$ or not can admits one or two generators.

Let $f: M \rightarrow R$ now be a $C^{\infty}$ Morse function. Let $\mathcal{D}(M)$ be a group of diffeomorphisms which preserve the Morse function [21] $f$ on $M$ (Möbius). Consider a group $H$ of automorphisms of critical sets $X_{i}$ on $M$ which are induced by the action of diffeomorphisms $h$ of a group $D(M)$ which preserve the Morse function $f$. 
In other words, the $h$ here are from the stabilizer $S(f) \triangleleft D(M)$. We note that the generators with stabilizers with the right action by diffeomorphisms $\pi_{0} S\left(\left.f\right|_{X_{i}}, \partial X_{i}\right)$ are $\tau_{i}$. The generators of the cyclic group $Z$ which define a shift are $\rho$. Since the group action is continuous, this implies that the $\rho$ can realize only cyclic shifts, else one would change the domains of of simple connectedness $X_{i}$ (critical sets) order.

The group $H \simeq \mathbb{Z} \ltimes(\mathbb{Z})^{n}=\langle\rho, \tau\rangle$ with defined above homomorphism in Aut $Z^{n}$ has two generators and non trivial relations [18] $\left\langle\rho, \tau_{1}, \ldots, \tau_{n} \mid \rho \tau_{i(\bmod n)} \rho^{-1}=\tau_{i+1(\bmod n)}, \tau_{i} \tau_{j}=\tau_{j} \tau_{i}, i, j \leq n\right\rangle$.

Corollary 4.1. A center of the group $H=\mathbb{Z} \ltimes_{\varphi}(\mathbb{Z})^{n}$ is a normal closure of sets: diagonal of $\mathrm{Z}^{n}$, trivial an element and subgroup that is kernel of action by conjugation of elements of $\mathrm{Z}^{n}$ that is $\left(\left\langle\rho^{2 n}\right\rangle \simeq 2 n \mathrm{Z}\right)$. In other words,

$$
Z(H)=\langle(1 ; \underbrace{h, h, \ldots, h}_{n}), e, 2 n \mathrm{Z} \ltimes \mathrm{E} \simeq 2 n \mathbb{Z} \times \mathbb{Z} .\rangle,
$$

where $h, g \in \mathrm{Z}$.

Proof. Since the action is defined by conjugation and relation $\rho^{2 n} \tau_{i} \rho^{-2 n}=\tau_{i}$ holds then the element $\left(\rho^{2 n}, e\right)$ commutates with every $\left(e, \tau_{i}\right)$. The stabilizer of such an action over the $\mathbb{Z}$-space $X=\left\{x_{1}, x_{2}, \ldots, x_{2 n}\right\}$ is the subgroup $2 n \mathrm{Z}$.

So subgroup stabilize all $x_{i}$ of $Z$-space $M$. Other words subgroup $\left\langle\rho^{2 n}\right\rangle$ belongs to kernel of action $\phi$. Besides the element $(1 ; \underbrace{h, h, \ldots, h}_{n})$ will not be changed by action of conjugation of any element from $H$ because any permutation elements coordinate of diagonal of $\mathrm{Z}^{n}$ does not change it.

Thus, $Z(H) \simeq 2 n \mathbb{Z} \times \mathbb{Z}$.

Corollary 4.2. The centre of a group of the form $\mathbb{Z} \ltimes_{\phi}(\mathcal{B})^{n} \simeq(\mathbb{Z}, X) \curlywedge \mathcal{B}$ generates, by normal closure of: center of diagonal of $\mathcal{B}^{n}$, trivial an element, and $n \mathrm{Z}_{X} \mathcal{E}$.

\section{Conclusion}

The minimal generating set for wreath-cyclic groups have been constructed. The investigation of structure of wreath product that described in book of Meldrum [11] was generalized on case of non-faithful group action of an active group. The center of wreath product, where active group action is non-faithfully. New estimations of the upper bound of generating set of commutator subgroup was obtained.

Acknowledgement: We thanks to Antonenko Alexandr for a graphical support, also we are grateful to Samoilovych I.

Conflicts of Interest: The author(s) declare that there are no conflicts of interest regarding the publication of this paper. 


\section{ReFERENCES}

[1] Laurent Bartholdi, Rostislav I Grigorchuk, and Zoran Šuni. Branch groups. In Handbook of algebra, volume 3, Elsevier, 2003, 989-1112.

[2] Agnieszka Bier and Vitaliy Sushchansky. Kaluzhnin's representations of sylow p-subgroups of automorphism groups of p-adic rooted trees. Algebra Discr. Math., 19 (2015), 19-38.

[3] Ievgen V Bondarenko. Finite generation of iterated wreath products. Arch. Math., 95 (4) (2010), 301-308.

[4] A. Woryna, The rank and generating set for iterated wreath products of cyclic groups, Commun. Algebra, 39 (7) (2011), 2622-2631.

[5] John Dixon and Brian Mortimer. Permutation groups, Springer Science \& Business Media, Volume 163, 1996.

[6] Andrea Lucchini. Generating wreath products and their augmentation ideals. Rend. Semin. Mat. Univ. Padova, 98 (1997), $67-87$.

[7] Yu V Dmitruk and VI Sushchanskii. Structure of sylow 2-subgroups of the alternating groups and normalizers of sylow subgroups in the symmetric and alternating groups. Ukr. Math. J., 33 (1981), 235-241.

[8] I. Martin Isaacs. Commutators and the commutator subgroup. Amer. Math. Mon., 9 (1977), 720-722.

[9] Léo Kaloujnine. Sur les $p$-groupes de sylow du groupe symétrique du degré $p^{m}$. C. R. Acad. Sci., 221 (1945), $222-224$.

[10] Yaroslav Lavrenyuk. On the finite state automorphism group of a rooted tree. Algebra Discr. Math., 2002 (2002), $79-87$.

[11] John DP Meldrum. Wreath products of groups and semigroups, volume 74. CRC Press, 1995.

[12] Alexey Muranov. Finitely generated infinite simple groups of infinite commutator width. Int. J. Algebra Comput., 17 (2007), 607-659.

[13] Volodymyr Nekrashevych. Self-similar groups, Mathematical Surveys and Monographs, Amer. Math. Soc., New York, Volume 117, 2005.

[14] Nikolay Nikolov. On the commutator width of perfect groups. Bull. Lond. Math. Soc., 36 (2004), 30-36.

[15] Vladimir Sharko. Smooth and topological equivalence of functions on surfaces. Ukr. Math. J., 55 (2003), 832-846.

[16] Ruslan Skuratovskii. Corepresentation of a sylow p-subgroup of a group s n. Cybern. Syst. Anal., 45(1) (2009), 25-37.

[17] Ruslan Skuratovskii. Minimal generating sets for wreath products of cyclic groups, groups of automorphisms of Ribe graph and fundumental groups of some Morse functions orbits. In Algebra, Topology and Analysis (Summer School), (2016), 121-123.

[18] Ruslan Skuratovskii. Minimal Generating Set and a Structure of the Wreath Product of Groups, and the Fundamental Group of the Orbit Morse Function. arXiv:1901.00061 [math.GR], (2019), 1-14.

[19] Ruslan Skuratovskii. Minimal generating sets of cyclic groups wreath product (in russian). In International Conference, Mal'tsev meetting, (2018), 118.

[20] Vitaly Ivanovich Sushchansky. Normal structure of the isometric metric group spaces of p-adic integers. Algebraic structures and their application. Kiev, (1988), 113-121.

[21] Sergiy Maksymenko. Deformations of functions on surfaces by isotopic to the identity diffeomorphisms. arXiv:1311.3347 [math.GT], (2013).

[22] James Wiegold. Growth sequences of finite groups. J. Aust. Math. Soc., 17(2)(1974), 133-141. 